\title{
Lymphangiogenesis in Gastric Cancer regulated through Akt/mTOR-VEGF-C/VEGF-D axis
}

\author{
Hongxia Chen ${ }^{1 \dagger}$, Runnian Guan ${ }^{2 \dagger}$, Yupeng Lei ${ }^{1 \dagger}$, Jianyong Chen ${ }^{3 \dagger}$, Qi Ge ${ }^{1}$, Xiaoshen Zhang ${ }^{4}$, Ruoxu Dou ${ }^{4}$, \\ Hongyuan Chen ${ }^{5}$, Hao Liu ${ }^{4}$, Xiaolong Qi ${ }^{1,4^{*}}$, Xiaodong Zhou ${ }^{1 *}$ and Changyan Chen ${ }^{1,6}$
}

\begin{abstract}
Background: Lymphangiogenesis plays a significant role in metastasis and recurrence of gastric cancer. There is no report yet focusing on the modulation of VEGF pathway and lymphangiogenesis of gastric cancer by targeting Akt/mTOR pathway. This study aims to demonstrate the relationship between Akt/mTOR pathway and VEGF-C/-D in gastric cancer.

Methods: We collected surgically resected gastric adenocarcinoma specimens from 55 consented patients. Immunohistochemistry staining of p-Akt, p-mTOR, VEGF-C, VEGF-D were performed and scored by two independent pathologists. The results were presented as staining intensity and positive staining cell rate. We also measured lymphatic vessel density (LVD) by D2-40 staining. Different dosages of p-Akt inhibitor LY294002 (12.5 $\mu \mathrm{M}, 25 \mu \mathrm{M}$, $50 \mu \mathrm{M})$ and p-mTOR inhibitor Rapamycin ( $25 \mathrm{nM}, 50 \mathrm{nM}, 100 \mathrm{nM})$ were given to gastric cancer cell line SGC-7901 in vitro. The inhibition rate of cell growth was tested by MTT at $24 \mathrm{~h}, 48 \mathrm{~h}$ and $72 \mathrm{~h}$, respectively and protein expressions of Akt, p-Akt, mTOR, p-mTOR, VEGF-C and VEGF-D were examined by Western blot.
\end{abstract}

Results: The positive staining rates of p-Akt, p-mTOR, VEGF-C and VEGF-D in 55 gastric cancer clinical specimens were $74.54 \%, 85.45 \%, 72.73 \%$ and 58.18\%. p-Akt and p-mTOR were positively correlated with VEGF-C and VEGF-D $(p<0.01)$. The LVD increased with incremental tendency of staining intensity of $p$-Akt, p-mTOR, VEGF-C and VEGF-D. LY294002 or Rapamycin significantly suppressed SGC-7901 cell growth and the inhibition rate was dose and time dependent $(p<0.001)$. In addition, the protein expression of $p$-Akt and $p$-mTOR were positively correlated with that of VEGF-C and VEGF-D $(p<0.05)$.

Conclusions: The level of LVD in gastric cancer specimens was significant higher than that of normal gastric tissue and was positively correlated with p-Akt, p-mTOR, VEGF-C and VEGF-D. Inhibition of p-Akt and p-mTOR, in vitro, decreased tumor cell VEGF-C and VEGF-D significantly. Therefore, we concluded that lymphangiogenesis of gastric cancer might be related to Akt/mTOR-VEGF-CNEGF-D axis.

Keywords: Lymphangiogenesis, Gastric cancer, Akt/mTOR, VEGF

\section{Background}

Gastric cancer is one of the most common malignant neoplasms worldwide [1-3]. Common treatment strategies for gastric cancer include surgery, radiotherapy, chemotherapy and targeted therapy $[4,5]$. Treatment failures include metastasis and recurrence after operation. The main metastatic routes of gastric cancer are direct invasion, vascular metastasis, lymphatic metastasis and

\footnotetext{
*Correspondence: qixiaolongmd@gmail.com; yfyzxd@163.com

tEqual contributors

'Department of Gastroenterology, The First Affiliated Hospital of Nanchang University, 17 Yongwaizheng Street, Nanchang 330006, China

Full list of author information is available at the end of the article
}

enterocoelia metastasis [3]. Among those, lymphatic metastasis is an increasingly important criterion in judging the prognosis of gastric carcinoma and lymphangiogenesis is recognized as a significant predictor for the prognosis of patients with gastric cancer [1,2]. In addition, the stomach cancer lymphatic metastasis is believed to be an earlier event compared to vascular metastasis, happening even in early stage gastric cancer $[3,4]$. Therefore, the International Union against Cancer took the lymphangiogenesis rate and the metastasis amount of the lymph node into the staging of gastric cancer in evaluating the prognosis $[2,5]$. Besides, even if lymphadenectomy and

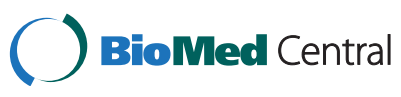

(c) 2015 Chen et al.; licensee BioMed Central. This is an Open Access article distributed under the terms of the Creative Commons Attribution License (http://creativecommons.org/licenses/by/4.0), which permits unrestricted use, distribution, and reproduction in any medium, provided the original work is properly credited. The Creative Commons Public Domain Dedication waiver (http://creativecommons.org/publicdomain/zero/1.0/) applies to the data made available in this article, unless otherwise stated. 
intraperitoneal chemotherapy were performed during lymph node negative progression, it also brought the positive effect on improving the 5-year survival rate of gastric cancer [5].

The two established ways of tumor cells entry into the lymprhatic system are invasion of existing lymphatic ducts and induction of lymphangiogenesis [3]. The latter is considered as the major way. In addition, lymphangiogenesis plays a decisive role in metastasis, recurrence and prognosis in early gastric cancer [4]. Thus, studies of lymphangiogenesis have a promising impact on the treatment. VEGF-C and VEGF-D were positively related to solid tumor lymphatic metastasis, lymphatic vessel density (LVD) and depth of tumor invasion and recurrence according to previous studies [6-8]. Besides, Akt/ mTOR pathway is also involved in the regulation of lymphangiogenesis and lymphatic metastasis [9-11]. Therefore, the relationship between Akt/mTOR pathway and VEGF pathways is worthy to be elucidated. To our knowledge, there is no report yet focusing on the modulation of VEGF pathway and lymphangiogenesis by targeting Akt/mTOR pathway in gastric cancer. In this study, we aim to demonstrate the relationship between Akt/mTOR pathway and VEGF-C/-D in gastric cancer.

\section{Methods}

\section{Clinical specimen}

Surgical resected gastric adenocarcinoma specimens were collected from 55 patients with gastric cancer from April 2011 to November 2012. All of them received no radiotherapy, chemotherapy or immunotherapy before the operation. The study was approved by ethics committee of the First Affiliated Hospital of Nanchang University and all participants gave written informed consent.

\section{Immunohistochemistry}

All tissue samples were fixed with $10 \%$ formalin, routinely embedded in paraffin, cut into 4- $\mu \mathrm{m}$ thick as serial sections, dewaxed with xylene, rehydration with an ethanol gradient and washed with distilled water. Antigen retrieval was performed by submerging sections in antigenic retrieval buffer (0.5 M EDTA), and microwaving for $20 \mathrm{~min}$. Sections were then treated with $3 \%$ hydrogen peroxide solution. The samples were blocked with $5 \%$ normal goat serum and then incubated overnight at $4{ }^{\circ} \mathrm{C}$ with the primary antibody: p-Akt (ser473) (Rabbit, 1:100, Cell Signaling Technology, USA), p-mTOR (ser2448) (Rabbit, 1:200, Abcam, USA), VEGF-C (Goat, 1:400, R\&D, USA), VEGF-D (Mouse, 1:50, Santa Cruz, USA) and D2-40 (Mouse, 1:100, ZSGB-BIO, China). Then, second antibody was added, and the samples were incubated for $30 \mathrm{~min}$ at room temperature and then developed with DAB. 5\% normal goat serum was used as a negative control instead of the primary antibody. Immunohistochemistry staining was scored by two independent pathologists. Results were presented in the form of staining intensity $(+/++/+++)$ and positive staining cell rate.

\section{Lymphatic vessel density}

According to Masakau criteria, D2-40 positive staining (single endothelial cell or cell clusters) was marked as positive microlymphatic vessel. The vascular-rich area in para-neoplastic location and tumor center were defined and 5 fields of "hot spots" were randomly counted under high magnification $(\times 400)$. The average was counted as the LVD.

\section{Cell survival}

Three different dosages $(12.5 \mu \mathrm{M}, 25 \mu \mathrm{M}, 50 \mu \mathrm{M})$ of Akt activation inhibitor LY294002 (Sigma) or mTOR activation inhibitor $(25 \mathrm{nM}, 50 \mathrm{nM}, 100 \mathrm{nM})$ Rapamycin (Sigma) were given to gastric cancer cell line SGC-7901. The inhibition rate of cell growth was tested by MTT assay at $24 \mathrm{~h}, 48 \mathrm{~h}$ and $72 \mathrm{~h}$, respectively.

\section{Western blot}

SGC-7901 cells were homogenized in RIPA lysis buffer (10 mM Tris-HCl, pH 7.4, 150 mM NaCl, 600 mM NP-40, $1 \%$ Triton X-100, 10\% glycerol, $1 \mathrm{mM}$ phenylmethylsulfonyl fluoride, $1 \mathrm{mM}$ sodium fluoride, and $1 \mathrm{mM}$ sodium orthovanadate) and put on ice for $2 \mathrm{~h}$. Protein concentration of each sample was determined by Bio-Rad Protein Assay kit (Bio-Rad, Laboratories, Hercules, CA, USA) according to the instruction of manufacturer. Lysates containing $50 \mu \mathrm{g}$ of protein were separated by SDS-PAGE and then transferred to nitrocellulose membranes. Nonspecific reactions were blocked for $4 \mathrm{~h}$ with TBS-T $(50 \mathrm{mM}$ Tris. $\mathrm{HCl}, \mathrm{pH} 7.4,150 \mathrm{mM} \mathrm{NaCl}, 0.1 \%$ Tween 20) containing $5 \%$ non-fat dry milk. Then membranes were incubated overnight at $4^{\circ} \mathrm{C}$ with primary antibody: Akt (Mouse, 1:2000, Cell Signaling Technology, USA), p-Akt (ser473) (Rabbit, 1:2000, Cell Signaling Technology, USA), mTOR (Rabbit, 1:1000, Cell Signaling Technology, USA), p-mTOR (ser2448) (Rabbit, 1:3000, Abcam, USA), VEGF-C (Goat, 1:1000, R\&D, USA) and VEGF-D (Mouse, 1:500, Santa Cruz, USA). After being washed with TBS-T containing non-fat dry milk, the membranes were incubated with horseradish peroxidase-conjugated secondary antibodies. The protein blots were visualized by chemiluminescence using ECL (Amersham).

\section{Statistical analysis}

Independent samples $\mathrm{T}$ test between two groups and single factor analysis of variance (ANOVA) for multiple comparisons were utilized when the data in accordance with normal distribution and homogeneity of variance. Otherwise, Mann-Whitney test between two groups and Kruskal-Wallis test among multi-groups were conducted. 
Spearman analysis was used to measure the correlation of measurement and count data. Statistical analyses were performed by SPSS17.0 (SPSS Inc., Chicago, IL, USA). A p-value of $<0.05$ was considered statistically significant.

\section{Results}

Expression of p-Akt, p-mTOR, VEGF-C and VEGF-D in situ The immunohistochemistry stainings of p-Akt, p-mTOR, VEGF-C and VEGF-D in 55 gastric cancer clinical specimens were shown (Figure 1). The positive staining presented mostly in cytoplasm and a few nuclear stained in $\mathrm{p}$-Akt and p-mTOR. The positivity rates of p-Akt, p-mTOR, VEGF-C and VEGF-D were $74.54 \%, 85.45 \%$, $72.73 \%$ and $58.18 \%$ respectively. The quantification of staining intensity was shown in Table 1.

The correlations of p-Akt and p-mTOR/VEGF-C/ VEGF-D, p-mTOR and VEGF-C/VEGF-D were further analyzed. p-Akt expression was positively correlated with $\mathrm{p}$-mTOR $(\mathrm{p}=0.001)$, VEGF-C $(\mathrm{p}=0.002)$ and VEGF-D $(\mathrm{p}=0.009)$ (Figure 1E). Besides, $\mathrm{p}-\mathrm{mTOR}$ was also significantly positive related to VEGF-C $(\mathrm{p}<0.001)$ and VEGF-D $(\mathrm{p}=0.003)$.

\section{Lymphatic vessel density}

D2-40 staining was used to calculate the LVD for gastric cancer (Figure 2A) and matched normal gastric tissue (Figure 2B). According to the results, D2-40 stained mainly in lymphatic endothelium in scattered or clustered distribution. Thin lymphatic vessel wall was lacking basal layer. The highest LVD was observed at the edge of tumor with a dense cluster-like morphology. In addition, D2-40 was expressed in the submucosa of normal tissue, with a weaker expression in the mucosa or muscle layer as well. The average LVD of gastric carcinoma and normal tissue

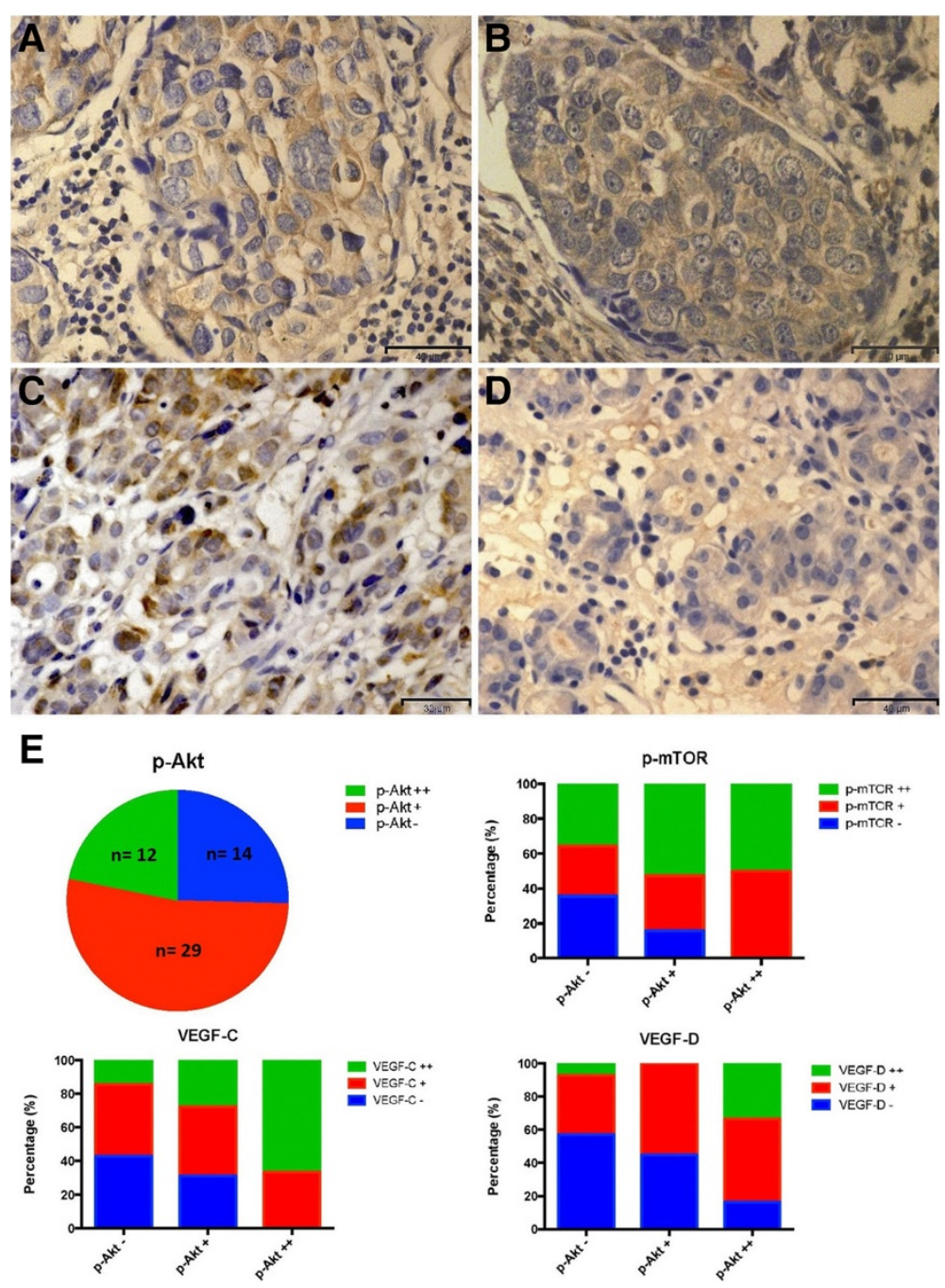

Figure 1 Immunohistochemistry staining of p-Akt, p-mTOR, VEGF-C and VEGF-D in gastric cancer specimen ( $\times 400)$. A: p-Akt, B: p-mTOR, C: VEGF-C, D: VEGF-D, E: The correlation between $p$-Akt and p-mTOR/NEGF-CNEGF-D. 
Table 1 Immunohistochemistry staining of p-Akt, p-mTOR, VEGF-C and VEGF-D in gastric cancer specimens

\begin{tabular}{lllll}
\hline & \multicolumn{2}{l}{ Intensity $(\mathbf{n})$} & & $\begin{array}{l}\text { Positive } \\
\text { rate (\%) }\end{array}$ \\
\cline { 2 - 4 } & - & + & ++ & \\
\hline p-Akt & 14 & 29 & 12 & 74.54 \\
p-mTOR & 8 & 21 & 26 & 85.45 \\
VEGF-C & 15 & 22 & 18 & 72.73 \\
VEGF-D & 23 & 27 & 5 & 58.18 \\
\hline
\end{tabular}

were $94.18 \pm 72.965$ vs $23.31 \pm 21.569$ number $/ 5$ high power fields, $\mathrm{p}<0.001$.

LVD were positively correlated with $\mathrm{p}$-Akt, p-mTOR, VEGF-C and VEGF-D expression per Spearman analysis $(p<0.05)$. LVD increased with the incremental tendency of staining intensity of p-Akt, p-mTOR, VEGF-C and VEGF-D (Figure 2C).

\section{Effects of LY294002 and Rapamycin on SGC-7901 cell growth}

SGC-7901 cells were cultured with three different dosages of LY294002 for $24 \mathrm{~h}, 48 \mathrm{~h}$ and $72 \mathrm{~h}$. According to MTT assay, SGC-7901 cell growth was curbed and the inhibition rate was significantly correlated with LY294002 dosage and action time $(\mathrm{p}<0.001)$ (Figure 3A).

Besides, cells were also cultured with different dosages of Rapamycin for $24 \mathrm{~h}, 48 \mathrm{~h}$ and $72 \mathrm{~h}$, respectively. The inhibition rate of SGC-7901 was also significantly correlated with either Rapamycin dosage or action time $(\mathrm{p}<0.001)$ (Figure 3B). Therefore, both LY294002 and

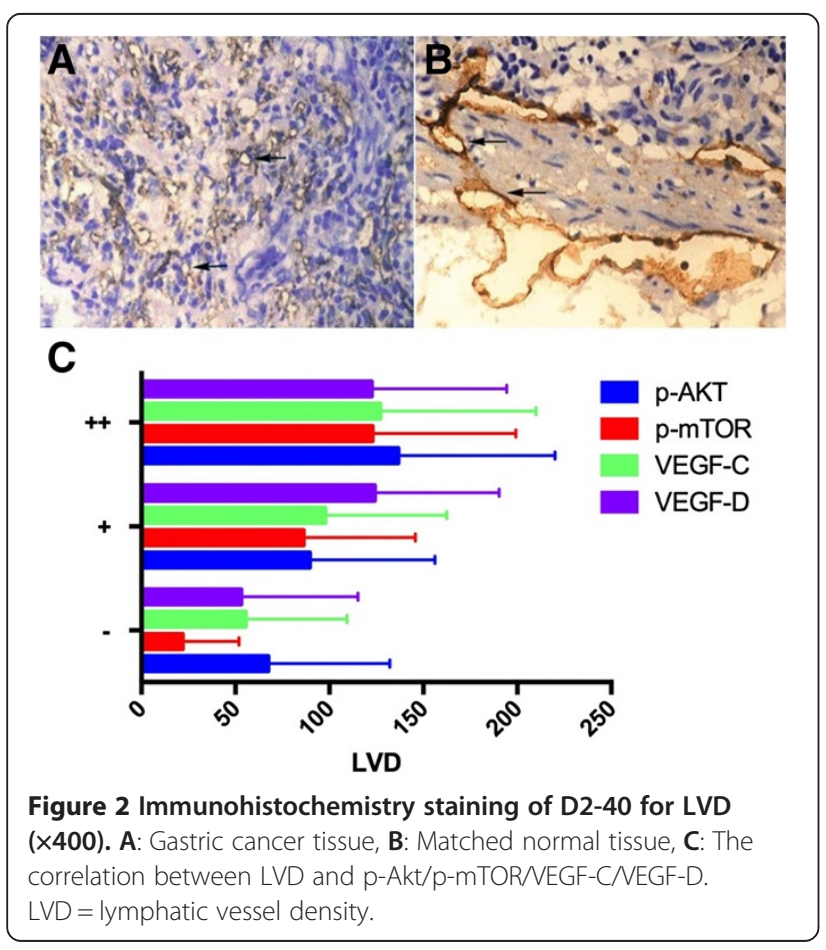

Rapamycin could inhibit the growth of SGC-7901 in either time-dependent or dose-dependent manner.

Effect of LY294002 on Akt/mTOR pathway and VEGF-C/-D The protein level of Akt, p-Akt, mTOR, p-mTOR, VEGF-C and VEGF-D in SGC-7901 with LY294002 $(50 \mu \mathrm{M})$ inhibition were examined by Western blot. Among them, p-Akt, p-mTOR, VEGF-C and VEGF-D were gradually decreased with the prolongation action time of LY294002 (Figure 3C). There was a significant difference when compared with the group without LY294002 ( $<<0.05)$. However, there was no statistical difference for Akt and mTOR $(p>0.05)$. By further conducting a dual variant relation analysis, $\mathrm{p}$-Akt was positively correlated with $\mathrm{p}-\mathrm{mTOR} / \mathrm{VEGF}-\mathrm{C} / \mathrm{VEGF}-\mathrm{D}$ $(\mathrm{p}<0.05)$ and $\mathrm{p}-\mathrm{mTOR}$ was also positively related to VEGF-C/VEGF-D $(\mathrm{p}<0.05)$.

Effect of Rapamycin on mTOR pathway and VEGF-C/-D The protein level of Akt, p-Akt, mTOR, p-mTOR, VEGF-C and VEGF-D in SGC-7901 with Rapamycin (100 nM) inhibition were examined by Western blot. Among them, p-mTOR, VEGF-C and VEGF-D expression were decreased with the prolongation action time of Rapamycin (Figure 3C). There was a significant difference when compared with the group without Rapamycin $(\mathrm{p}<0.05)$. However, there was no statistical difference for Akt, pAkt and mTOR ( $p>0.05$ ). By further conducting a dual variant relation analysis, $\mathrm{p}-\mathrm{mTOR}$ was positively correlated with VEGF-C and VEGF-D $(\mathrm{p}<0.05)$.

\section{Discussion}

Akt/mTOR signaling pathway is an important intracellular pathway, which responds to cytokine signals, and is proved to play a major role in cell growth, metabolism and apoptosis [12,13]. Akt/mTOR pathway involves in various pathological mechanisms of colorectal cancer, gastric cancer, liver cancer, breast cancer and uterine cancer etc. [12-14]. Our previous study confirmed that p-Akt involved in angiogenesis of gastric adenocarcinoma and Akt activation may contribute to angiogenesis via VEGF-A up-regulation [15]. In this study, the positive staining rates of p-Akt and p-mTOR in 55 cases of gastric cancer were $74.54 \%$ and $85.45 \%$ respectively. Besides, p-Akt was positively correlated with p-mTOR $(\mathrm{p}<0.01)$. This result was consistent with previous studies and confirmed the significant role of Akt/mTOR pathway in gastric cancer. The clinical prognosis of gastric cancer is also proposed to be associated with Akt pathway and VEGF pathway. For instance, Murakami et al. suggested that the prognosis of gastric cancer patients without p-Akt expression was always better than p-Akt positive ones, and the 5-year survival rates were $68 \%$ and $35 \%$ for patients with T3 or T4 stage 

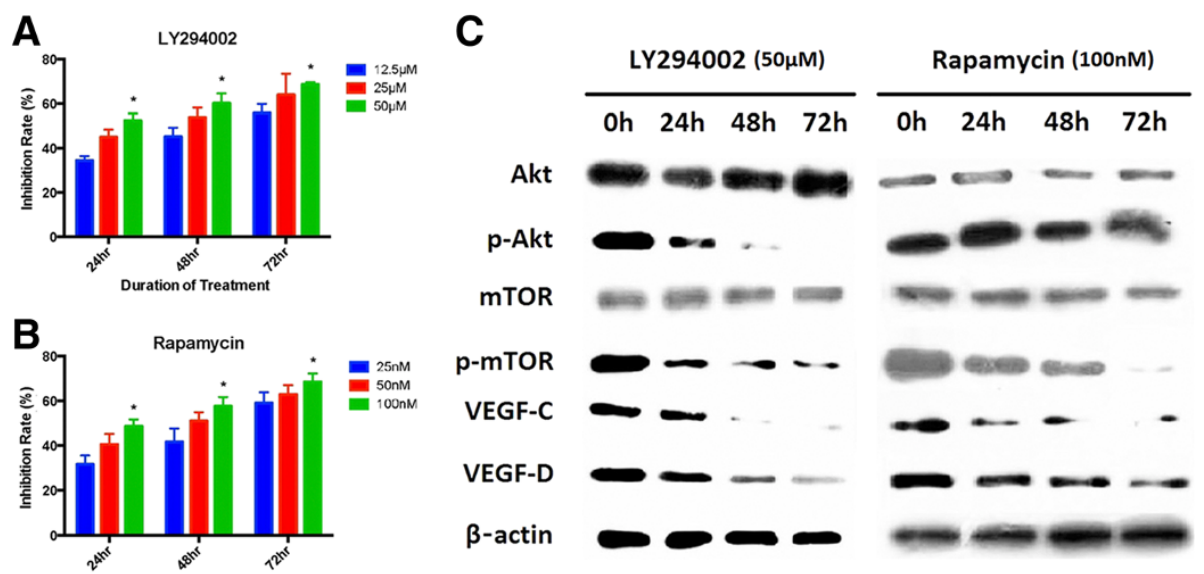

Figure 3 Inhibition of p-Akt and p-mTOR in SGC-7901 cells by LY294002 and Rapamycin. A: SGC-7901 cell growth with intervention of different time points and dosages of LY294002, B: SGC-7901 cell growth with intervention of different time points and dosages of Rapamycin, C: Effect of LY294002 (50 HM) and Rapamycin (100 nM) on Akt/mTOR pathway and VEGF-C/-D by Western blot.

tumors, respectively [16]. Besides, the Akt encoding gene PI3CA of upstream protein PI3K was associated with disease-free survival [17]. In addition, Wang et al. indicated that VEGF-C, VEGF-D and LVD of gastric cancer were related to tumor size and overall survival according to a study in 123 cases $[18,19]$. The correlation between VEGF-C/LVD and prognosis in tumor differentiation, $\mathrm{T}$ stage, lymph node metastasis, and distant metastasis were also confirmed [20]. Another study further suggested using the combination of VEGF-C/-D and CA199 to predict lymphatic vessel metastasis [21].

Given that Akt/mTOR pathway and VEGF-C/-D play a vital role in lymphatic metastasis and prognosis, our study aims to explore the relationship between two pathways and further promote the mechanism of lymphangiogenesis and metastasis. According to the results of staining in situ, p-Akt and p-mTOR were positively correlated with VEGF-C and VEGF-D $(\mathrm{p}<0.01)$. In terms of LVD, D2-40 level of gastric carcinoma and normal tissues were examined at the same time. LVD in gastric cancer was four times higher than that of normal tissue. Besides, LVD was significantly correlated with p-Akt, p-mTOR, VEGF-C and VEGF-D $(\mathrm{p}<0.05)$. Notably, LVD as well as VEGF-C/-D was obviously lower in patients with negative expression of p-Akt and p-mTOR than positive ones. It suggested that both Akt/mTOR pathway and VEGF pathway involved in lymphangiogenesis and lymphatic vessel metastasis in gastric cancer. Similar results were also confirmed by other studies. $Y u$ et al. suggested that p-mTOR were highly consistent with lymph node metastasis and could be an independent predictor of lymphatic metastasis and prognosis of gastric cancer [22]. Besides, Onogawa et al. indicated that VEGF-C was highly correlated with lymphatic metastasis according to a study conducted in 140 cases of early gastric cancer [23]. VEGF-C as well as
VEGF-D was also regarded as a predictor of lymphatic metastasis in patients with early gastric cancer [24]. In the study, we further confirmed the relationship of Akt/ mTOR, VEGF-C/-D and LVD in gastric cancer in situ.

LY294002, as an Akt activation inhibitor, is confirmed with the anti-tumor effect and promoted the apoptosis of gastric cancer in vitro and in vivo [25]. Rapamycin known as an mTOR activation inhibitor is clinically used for its anti-tumor effect and immunomodulation [26]. The results of our study further verified that LY294002 and Rapamycin could efficiently inhibit the growth of SGC-7901 cells. Besides, LY294002 at $50 \mu \mathrm{M}$ ) and Rapamycin at $100 \mathrm{nM}$ had the most effective inhibition rate of $(52.53 \pm 3.19) \%$ and $(48.72 \pm 3.06) \%$ at $24 \mathrm{~h}$. When prolonging to $72 \mathrm{~h}$, the inhibition rates were $(68.76 \pm 1.00) \%$ and $(68.50 \pm 3.77) \%$ respectively. Then, we observed the influence of phosphorylation inhibition of Akt and mTOR on lymphatic factors, VEGF-C and VEGF-D. High concentration of LY294002 $(50 \mu \mathrm{M})$ and Rapamycin $(100 \mathrm{nM})$ were used for the intervention study. According to the results, Rapamycin suppressed the expression of p-mTOR and LY294002 efficiently inhibited the expression of p-Akt as well as p-mTOR. It is noted that both inhibitors could efficiently inhibit the expression of VEGF-C/-D. Besides, the down-regulation of p-Akt/p-mTOR showed a high consistency with that of VEGF-C/-D. Therefore, Akt/mTOR pathway was proposed to be closely related to VEGF-C/-D. The similar results were also previously reported in other tumor models. For example, Zhang et al. decreased the insulin receptor by short hairpin RNA in cancer cell lines, LCC6 and T47D. The cell proliferation, angiogenesis, lymphangiogenesis and metastasis were also inhibited due to the down-regulation of $\mathrm{p}$-Akt and inactivation of VEGF-D [27]. Besides, mTOR inhibition could suppress 
the expression of VEGF-C and effectively reduce lymphangiogenesis in pancreatic cancer mouse model [11]. In another study, VEGF-D was degraded by Honokiol to inhibit lymphangiogenesis of lung cancer xenograft [28]. IL-6 could also regulate VEGF-C at mRNA level through Akt/mTOR signaling pathway, which effectively decreased the lymphangiogenesis and metastasis in oral squamous cell carcinoma [29].

\section{Conclusion}

The level of LVD in gastric cancer specimens was significant higher than that of normal gastric tissue and positively correlated with immunohistochemistry staining of p-Akt, p-mTOR, VEGF-C and VEGF-D. With the inhibition of p-Akt and p-mTOR in vitro, VEGF-C and VEGF-D were also significantly decreased. Therefore, we propose that lymphangiogenesis of gastric cancer might be efficiently modulated through Akt/mTOR-VEGF-C/ VEGF-D axis.

\section{Abbreviation}

LVD: Lymphatic vessel density.

\section{Competing interests}

The authors declare that they have no competing interests.

\section{Authors' contributions}

Study concepts and design: XZhou, HC; Data acquisition: HC, RG, HC; Data analysis: YL, JC; Manuscript preparation: QG, RD, XZhang; Manuscript revision: $X Q, H L, C C$. All authors have participated sufficiently in the study and approved the final version.

\section{Acknowledgement}

The study was supported by funding from National Science Foundation Grants of China (No. 81160307); Jiangxi Science \& Technology Pillar Program and the Science Foundation for Young Scholars of Jiangxi Province (No. 2007GQY1167); Voyage Project of Jiangxi Province Science and Technology Association.

\section{Author details \\ 'Department of Gastroenterology, The First Affiliated Hospital of Nanchang University, 17 Yongwaizheng Street, Nanchang 330006, China. ${ }^{2}$ Department of Gastroenterology, Kaiping Central Hospital, Kaiping 529300, China. ${ }^{3}$ Department of Gastroenterology, Jiangxi Provincial People's Hospital, Nanchang 330006, China. ${ }^{4}$ Department of Radiation Oncology, Massachusetts General Hospital and Harvard Medical School, Boston, MA, USA. ${ }^{5}$ Department of Pathogen Biology and Immunology, School of Basic Course, Guangdong Pharmaceutical University, Guangzhou 510060, China. ${ }^{6}$ Center for Drug Discovery, Northeastern University, Boston, MA 02115, USA.}

Received: 4 November 2014 Accepted: 20 February 2015 Published online: 07 March 2015

\section{References}

1. Siegel R, Ma J, Zou Z, Jemal A. Cancer statistics, 2014. CA Cancer J Clin. 2014;64:9-29.

2. Yoo YA, Kang MH, Lee HJ, Kim BH, Park JK, Kim HK, et al. Sonic hedgehog pathway promotes metastasis and lymphangiogenesis via activation of Akt, EMT, and MMP-9 pathway in gastric cancer. Cancer Res. 2011;71:7061-70.

3. Liu C, Lu P, Lu Y, Xu H, Wang S, Chen J. Clinical implications of metastatic lymph node ratio in gastric cancer. BMC Cancer. 2007;7:200

4. Brar SS, Mahar AL, Helyer LK, Swallow C, Law C, Paszat L, et al. Processes of care in the multidisciplinary treatment of gastric cancer: results of a RAND/UCLA expert panel. JAMA Surg. 2014;149:18-25.
5. Cunningham D, Chua YJ. East meets west in the treatment of gastric cancer. N Engl J Med. 2007;357:1863-5.

6. Karnezis T, Shayan R, Caesar C, Roufail S, Harris NC, Ardipradja K, et al. VEGF-D promotes tumor metastasis by regulating prostaglandins produced by the collecting lymphatic endothelium. Cancer Cell. 2012;21:181-95.

7. Ko YH, Jung CK, Lee MA, Byun JH, Kang JH, Lee KY, et al. Clinical significance of vascular endothelial growth factors (VEGF)-C and -D in resected non-small cell lung cancer. Cancer Res Treat. 2008:40:133-40.

8. He Y. Vascular endothelial cell growth factor receptor 3-mediated activation of lymphatic Eendothelium is crucial for tumor cell entry and spread via lymphatic vessels. Cancer Res. 2005;65:4739-46.

9. Chen H, Li L, Wang S, Lei Y, Ge Q, Lv N, et al. Reduced miR-126 expression facilitates angiogenesis of gastric cancer through its regulation on VEGF-A. Oncotarget. 2014;5:11873-5.

10. Zhou F, Chang Z, Zhang L, Hong YK, Shen B, Wang B, et al. Akt/Protein kinase $B$ is required for lymphatic network formation, remodeling, and valve development. Am J Pathol. 2010;177:2124-33.

11. Kobayashi S, Kishimoto T, Kamata S, Otsuka M, Miyazaki M, Ishikura H. Rapamycin, a specific inhibitor of the mammalian target of rapamycin, suppresses lymphangiogenesis and lymphatic metastasis. Cancer Sci. 2007;98:726-33.

12. Scartozzi M, Giampieri R, Maccaroni E, Mandolesi A, Biagetti S, Alfonsi S, et al. Phosphorylated AKT and MAPK expression in primary tumours and in corresponding metastases and clinical outcome in colorectal cancer patients receiving irinotecan-cetuximab. J Transl Med. 2012;10:71.

13. Heikkinen $T$, Greco D, Pelttari LM, Tommiska J, Vahteristo $P$, Heikkilä $P$, et al. Variants on the promoter region of PTEN affect breast cancer progression and patient survival. Breast Cancer Res. 2011;13:R130.

14. Xu W, Huang JJ, Cheung PC. Extract of pleurotus pulmonarius suppresses liver cancer development and progression through inhibition of VEGF-induced PI3KJAKT signaling pathway. PLoS One. 2012;7:e34406.

15. Zhou XD, Chen HX, Guan RN, Lei YP, Shu X, Zhu Y, et al. Protein kinase B phosphorylation correlates with vascular endothelial growth factor $\mathrm{A}$ and microvessel density ingastric adenocarcinoma. J Int Med Res. 2012:40:2124-34.

16. Murakami D, Tsujitani S, Osaki T, Saito H, Katano K, Tatebe S, et al. Expression of phosphorylated Akt (pAkt) in gastric carcinoma predicts prognosis and efficacy of chemotherapy. Gastric Cancer. 2007;10:45-51.

17. Shi J, Yao D, Liu W, Wang N, Lv H, Zhang G, et al. Highly frequent PIK3CA amplification is associated with poor prognosis in gastric cancer. BMC Cancer. 2012:12:50.

18. Wang XL, Fang JP, Tang RY, Chen XM. Different significance between intratumoral and peritumoral lymphatic vessel density in gastric cancer: a retrospective study of 123 cases. BMC Cancer. 2010;10:299.

19. Han FH, Li HM, Zheng DH, He YL, Zhan WH. The effect of the expression of vascular endothelial growth factor (VEGF)-C and VEGF receptor-3 on the clinical outcome in patients with gastric carcinoma. Eur J Surg Oncol. 2010;36:1172-9.

20. Wang TB, Deng MH, Qiu WS, Dong WG. Association of serum vascular endothelial growth factor-C and lymphatic vessel density with lymph node metastasis and prognosis of patients with gastric cancer. World J Gastroenterol. 2007;13:1794-7.

21. Tsirlis TD, Kostakis A, Papastratis G, Masselou K, Vlachos I, Papachristodoulou A, et al. Predictive significance of preoperative serum VEGF-C and VEGF-D, independently and combined with Ca19-9, for the presence of malignancy and lymph node metastasis in patients with gastric cancer. J Surg Oncol. 2010;102:699-703.

22. Yu G, Wang J, Chen Y, Wang X, Pan J, Li G, et al. Overexpression of phosphorylated mammalian target of rapamycin predicts lymph node metastasis and prognosis of chinese patients with gastric cancer. Clin Cancer Res. 2009;15:1821-9.

23. Onogawa S, Kitadai Y, Amioka T, Kodama M, Cho S, Kuroda T, et al. Expression of vascular endothelial growth factor (VEGF)-C and VEGF-D in early gastric carcinoma: correlation with clinicopathological parameters. Cancer Lett. 2005;226:85-90.

24. Arigami T, Natsugoe S, Uenosono $Y$, Yanagita S, Ehi $\mathrm{K}$, Arima H, et al. Vascular endothelial growth factor-C and -D expression correlates with lymph node micrometastasis in pNO early gastric cancer. J Surg Oncol. 2009;99:148-53.

25. Shin JY, Kim JO, Lee SK, Chae HS, Kang JH. LY294002 may overcome 5-FU resistance via down-regulation of activated p-AKT in Epstein-Barr virus-positive gastric cancer cells. BMC Cancer. 2010;10:425. 
26. Yao C, Liu J, Shao L. Rapamycin inhibits the proliferation and apoptosis of gastric cancer cells by down regulating the expression of survivin. Hepatogastroenterology. 2011;58:1075-80.

27. Zhang H, Fagan DH, Zeng X, Freeman KT, Sachdev D, Yee D. Inhibition of cancer cell proliferation and metastasis by insulin receptor downregulation. Oncogene. 2010;29:2517-27.

28. Wen J, Fu AF, Chen LJ, Xie XJ, Yang GL, Chen XC, et al. Liposomal honokio inhibits VEGF-D-induced lymphangiogenesis and metastasis in xenograft tumor model. Int J Cancer. 2009;124:2709-18.

29. Shinriki S, Jono H, Ueda M, Ota K, Ota T, Sueyoshi T, et al. Interleukin-6 signaling regulates vascular endothelial growth factor- $C$ synthesis and lymphangiogenesis in human oral squamous cell carcinoma. J Pathol. 2011;225:142-50

\section{Submit your next manuscript to BioMed Central} and take full advantage of:

- Convenient online submission

- Thorough peer review

- No space constraints or color figure charges

- Immediate publication on acceptance

- Inclusion in PubMed, CAS, Scopus and Google Scholar

- Research which is freely available for redistribution 